\title{
CONTAMINACIÓN DE CORRIENTES SUPERFICIALES POR TÓXICOS MINERO-METALÚRGICOS EN LA REGIÓN HIDROLÓGICA SONORA-SUR
}

\author{
Contamination of surface currents by mining-metalurgical toxics \\ in the South-Sonora hydrological region
}

EPISTEMUS

ISSN: 2007-8196 (electrónico)

ISSN: 2007-4530 (impresa)

Carlos E. Peña-Limón 1

Jonathan Parades-Aguilar ${ }^{2}$

Recibido: 14 de octubre de 2019,

Aceptado: 13 de noviembre de 2019

Autor de Correspondencia:

Dr. Carlos E. Peña Limón

Correo:carlos.pena@unison.mx

\section{Resumen}

La actividad minera genera enormes cantidades de materiales peligrosos que suelen llegar a aguas superficiales. Sonora, al poseer grandes minas activas e inactivas a las cercanías de ríos, presenta un riesgo potencial de contaminación en sus corrientes superficiales. El objetivo del presente estudio fue identificar las minas que pudieran presentar un mayor riesgo de contaminación a las corrientes superficiales de la Región Hidrológica Sonora-Sur utilizando una modificación de la metodología de la EPA (1991) para realizar evaluaciones preliminares de riesgo. Las minas identificadas con mayor riesgo fueron 1) En la cuenca Río Sonora, Buenavista del Cobre, Cerro Prieto, Mercedes, Santa Elena, y la mina inactiva Amelia, 2) En la cuenca Río Yaqui: La India, Mulatos, La Bonancita, La Caridad, Luz del Cobre y las minas inactivas Pilares y Cumobabi.

Palabras clave: Evaluación preliminar, Cuenca Río Sonora, Cuenca Río Yaqui.

\begin{abstract}
Mining use and produce large amounts of hazardous substances that often reach surface waters streams. Sonora, been a Mexican state with very large active and inactive mines located near the rivers, presents a high potential for contamination of its water surface bodies. The purpose of this study was to identify those mines with more possibilities to contaminate the surface water currents of the South-Sonora hydrological region using a modification of the EPA's methodology to carry out Preliminary Risk Assessment (EPA, 1991). The mines that represent higher risks were: 1) in the Sonora River Basin, "Buenavista del Cobre", "Cerro Prieto", "Mercedes", "Santa Elena" and the inactive mine "Amelia"; 2) In the Yaqui River Basin are "La India", "Mulatos", "La Bonancita", "Luz del Cobre", "La Caridad" and the abandoned mines "Pilares" and "Cumobabi".
\end{abstract}

Keywords: Preliminary risk assessment, Sonora river basin, Yaqui river basin.

1 Universidad de Sonora, México. Correo: carlos.pena@unison.mx

2 Universidad de Sonora, México.Correo:jonathan.parades@gmail.com 


\section{INTRODUCCIÓN}

Sonora con una superficie de 179355 km2, es considerado el estado líder en producción minerometalúrgica del país, ya que tiene en explotación grandes yacimientos de minerales produciendo cobre, metales preciosos, grafito, antracita, barita, cal, cemento, wollastonita etc. Las minas de cobre más importantes son "Buenavista del Cobre" (Municipio de Cananea) y "La Caridad", (Los municipios de Nacozari y Villa Hidalgo). Otras importantes minas de cobre son Mariquita, Milpillas, Luz del Cobre y Piedras Verdes. En Sonora se tienen también importantes yacimientos de oro siendo los principales explotados por las minas Mulatos, La India, El Chanate, Álamo Dorado, Las Mercedes, Noche Buena, SoledadDipolo, entre otras [1].

Por otra parte, en Sonora se localizan cinco regiones hidrológicas: RH-7 (Rio Colorado), RH-8 (Sonora-Norte), $\mathrm{RH}-9$ (Sonora-Sur), $\mathrm{RH}-10$ (Sinaloa) y $\mathrm{RH}-34$ (Cuencas Cerradas del Norte). De estas regiones, la más importante es la Región Hidrológica Sonora-Sur, que representa aproximadamente el $50 \%$ del área de Sonora (cubre una superficie de 117,363 km2 entre Sonora y Chihuahua). En esta región se encuentran la Cuenca Rio Yaqui (CRY) y la Cuenca Río Sonora (CRS), por donde fluye el 76\% del volumen total de precipitado al año y el $82 \%$ del escurrimiento total en el estado. Drenan a presas que abastecen de agua potable a gran parte de la población sonorense [2].

Por la CRS escurren los Ríos Sonora, San Miguel y Zanjón los cuales terminan en las presas "El Molinito" y "Abelardo Rodríguez" y por otra parte en la CRY, la corriente superficial más importante es el Río Yaqui que recibe diferentes nombres en su trayectoria. En esta cuenca están ubicadas las presas "La Angostura", “El Novillo" y “El

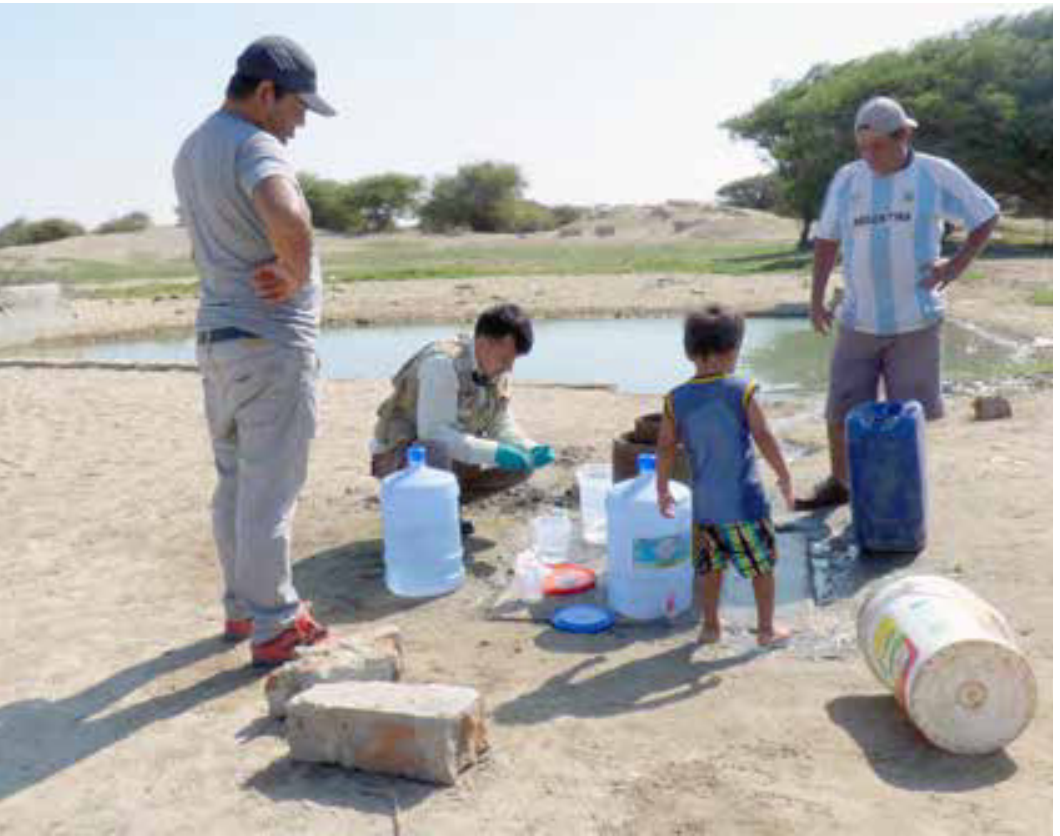

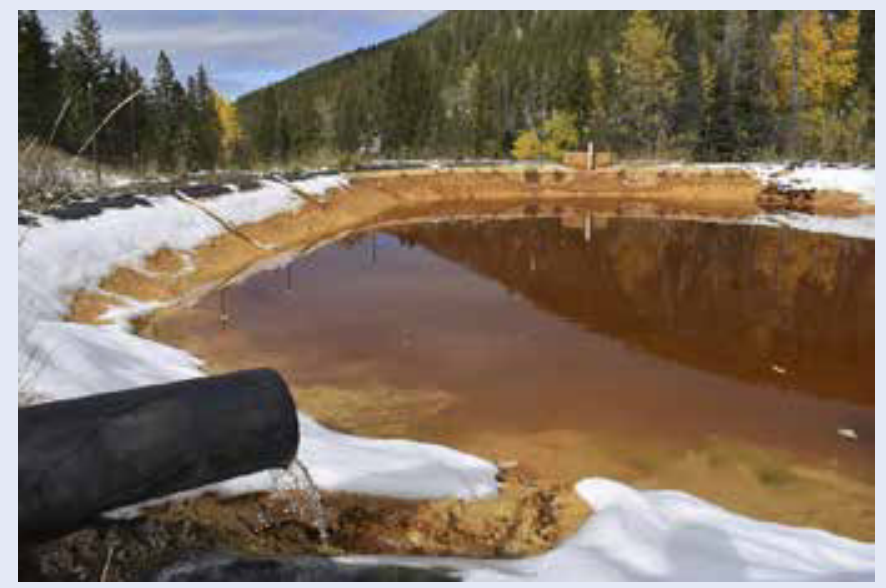

Oviachic" que además de servir de fuente de agua potable son usadas para generar electricidad y para alimentar canales de riego [2] (Figura 1).

La Ley de Aguas Nacionales vigente hace múltiples referencias a la calidad del agua, sin embargo, la gestión de la calidad de este recurso es insuficiente [3].

Al ser el estado de Sonora un importante estado minero, $y$ al situarse las minas generalmente a las cercanías de corrientes de agua, la sociedad sonorense percibe como un problema ambiental muy importante la posible contaminación de aguas superficiales por la actividad minero-metalúrgica en la región principalmente debido a derrames de:

- Insumos. Cianuro, usado principalmente en la extracción de oro.

- Subproductos o líquidos de procesos. Ácido sulfúrico durante su transporte y embarque, líquidos generados por lixiviación bacteriana de sulfuros, que frecuentemente se almacenan en presas construidas en cauces naturales.

- Residuos de minas. Drenaje ácido generado por la oxidación natural de sulfuros y/o la generación y acumulación de terreros de minerales sin valor (jales). Esta acumulación de desechos ocurre también en minas inactivas y suele llegar a contaminar las aguas superficiales [4].

Entre los contaminantes comunes provenientes de la actividad minera se encuentran los llamados "metales pesados", que son el propio mineral de interés, compuestos que lo acompañan o están involucrados en el proceso de extracción. Su impacto ecológico se debe principalmente a su alta toxicidad y a que son resistentes a la degradación [5]. Entre los principales metales contaminantes derivados de la actividad minera se encuentran: hierro, cobre, manganeso, zinc, plomo, níquel, cadmio, mercurio, selenio y arsénico [5]. La exposición a metales pesados produce diversas respuestas tóxicas (son cancerígenos y además producen daños en hígado, sistema nervioso y sistema reproductivo) [6]. 


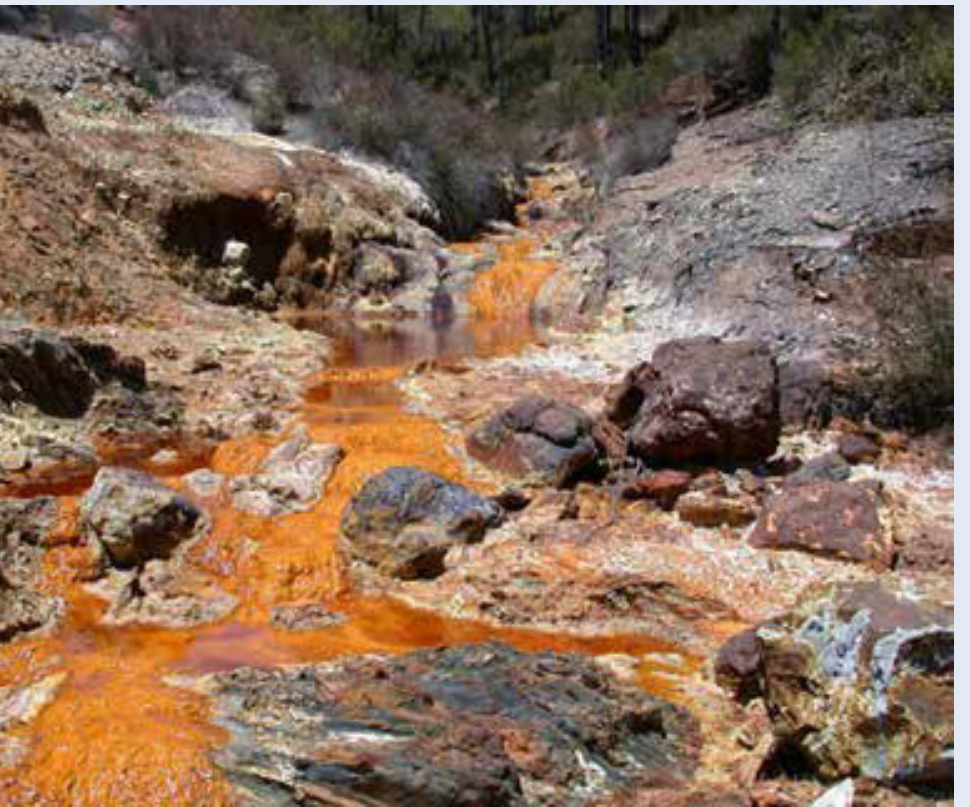

El propósito de este estudio fue identificar los sitios mineros existentes en la CRS y CRY de la RH-9 que más probablemente puedan contaminar las corrientes superficiales y descartar el estudio de aquellos que su peligrosidad es despreciable desde el punto de vista de la evaluación de riesgos.

\section{METODOLOGÍA}

Se obtuvo información en la Dirección General de Minería del Estado de Sonora y por consulta bibliográfica de estudios ambientales sobre las minas activas e inactivas con el propósito de identificar los sitios con fuentes de tóxicos que es posible que se liberen a las corrientes superficiales de las CRS y CRY de la RH-9. A este conjunto de datos se le denominó Archivo de Sitios Identificados.

Después, se realizó una evaluación preliminar en cada uno de los sitios identificados tomando como referencia la "Guía para realizar evaluaciones preliminares" (EP) de la EPA (1991), metodología que consiste en identificar y distinguir aquellos sitios que no pudieran causar daño a la salud de las poblaciones humanas y al medio ambiente utilizando solamente información existente [7].

Se modificó de la siguiente forma el método mencionado debido a que el propósito no fue identificar sitios que produzcan riesgos a la salud humana (RSH) sino identificar sitios con actividades minero-metalúrgicas que produzcan riesgos de contaminaciòn de una corriente superficial. Las modificaciones fueron las siguientes:

1.En la Categoría de Factor Blanco (B), se estableció únicamente el valor (5) tomando en cuenta que el recurso agua fue el único blanco de análisis y no se tomó como blanco ninguna población.

2. Para la categoría de factor "Sospecha de liberación" (PL) se asignó un valor de 550 para todas las minas, debido a que las minas seleccionadas para su análisis fueron aquellas en las que se sospecha que se liberan tóxicos a las corrientes superficiales.

3. Para la categoría de "Características de los residuos" (WC) se asignaron como valores 18,32 y 100 tomando en cuenta el valor de producción de cada mina y la magnitud del área afectada.

La ecuación para calcular la Ponderación de Peligro (PP) quedó:

$$
\mathbf{P P}=\frac{(\boldsymbol{P L})(\boldsymbol{B})(\boldsymbol{W C})}{2750}
$$

Los valores que se obtuvieron para la PP fueron 18,32 y 100 y se consideró que las que tuvieron un valor PP menor a 32 son las que no presentan riesgo de contaminación a las corrientes superficiales de las cuencas y no es necesario analizarlas.

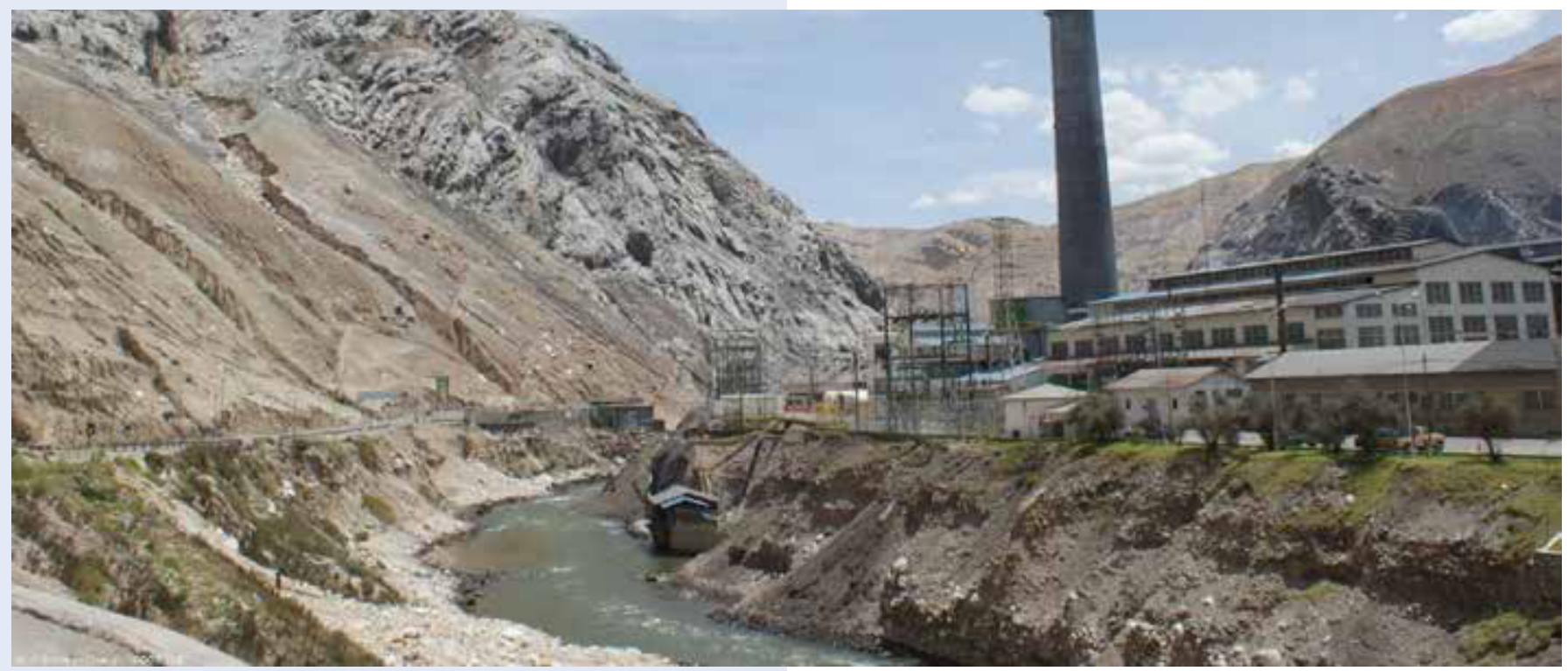




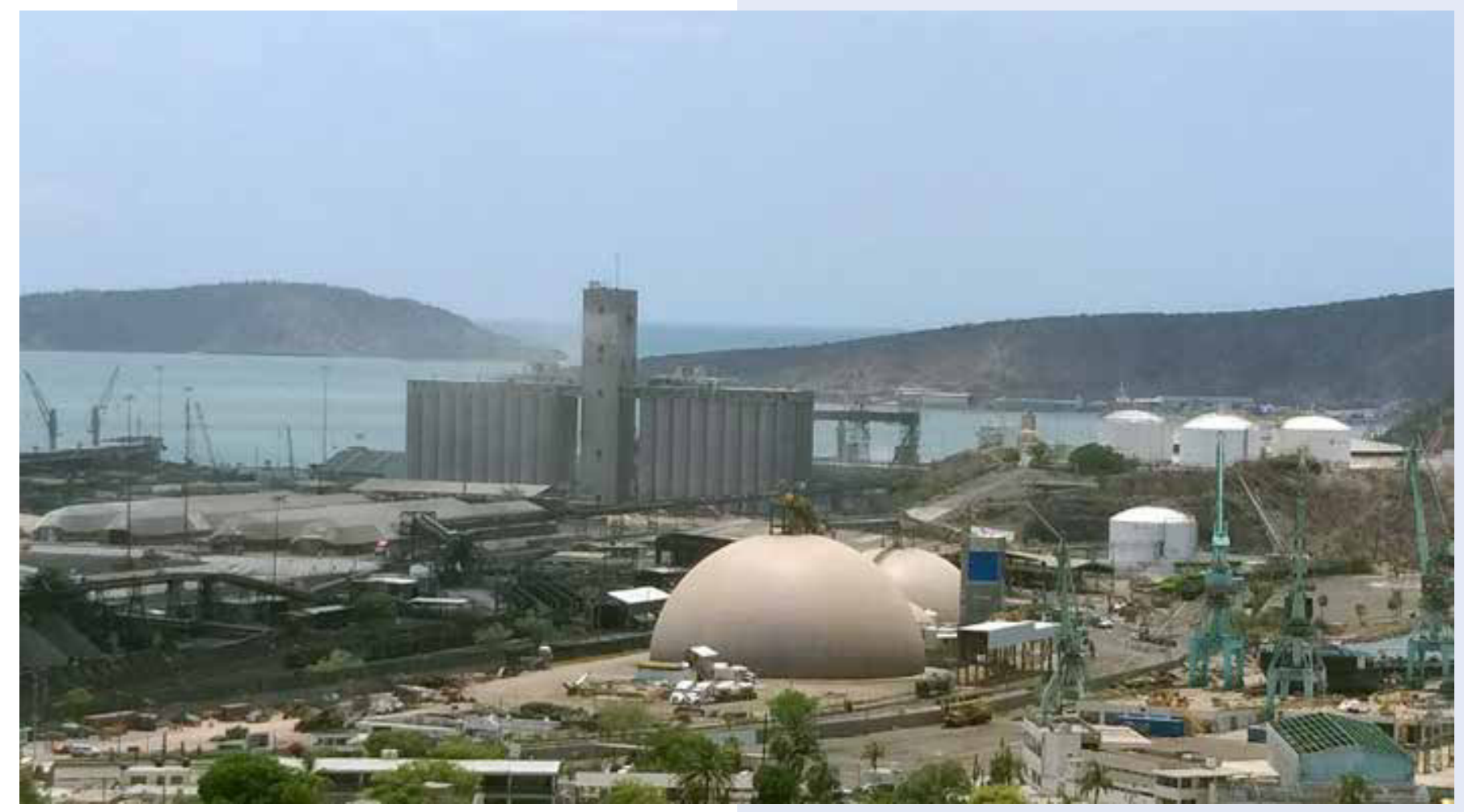

\section{RESULTADOS Y DISCUSIÓN}

Se identificaron todos los sitios minero-metalúrgicos de las CRS y CRY de la RH-9 y se observó que la mayoría de las minas activas e inactivas están ubicadas a las cercanías de los ríos (Figura 1).

La revisión bibliográfica arrojó la siguiente información:

1.Los principales minerales extraídos de esta zona son cobre y oro [1].

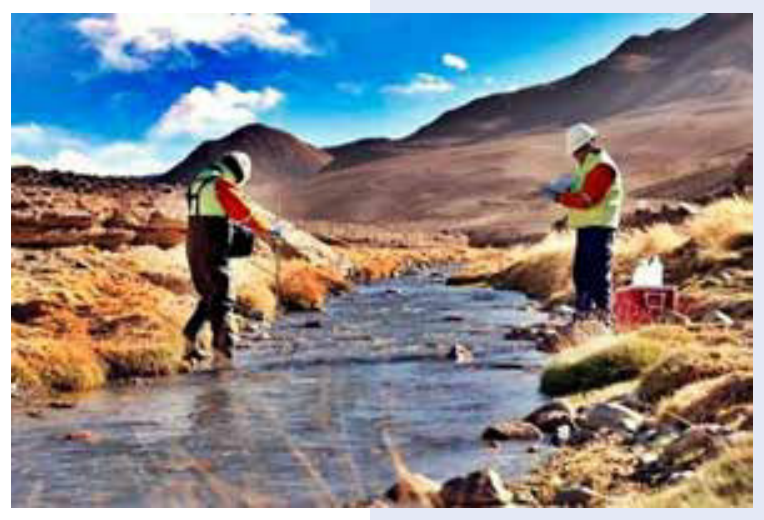

niveles de los mismos metales pesados en sedimentos de los ríos Sonora y Bacanuchi. Las posibles liberaciones de materiales peligrosos por la mina de Cananea pueden provenir principalmente de las presas de lixiviados (desastre ambiental ampliamente documentado producido por la liberación de 40,000 m3 de líquidos ácidos de las presas de lixiviación construidas en el cauce del

2. El minado a cielo abierto es el que se usa en las minas de mayor tamaño [1].

3. La metodología de beneficio es el lixiviado y tostación de concentrados [1].

4. Las contaminaciones ambientales de origen minero que más se han estudiado son las provocadas por la mina Buenavista del Cobre de la ciudad de Cananea. GómezÁlvarez et al. (2004) [8], así como Villalba-Atondo y Gómez-Álvarez (2011) [9] encontraron en agua y sedimentos en zonas impactadas de los ríos San Pedro y Bacanuchi metales pesados como cadmio, cobre, plomo, hierro, manganeso, níquel y zinc que excedieron los valores máximos permisibles establecidos por la Norma Oficial Mexicana. Estos valores además se relacionaron directamente con los vertimientos e infiltraciones de desechos ácidos provenientes de la Concentradora Vieja. León-García et al. (2018) [4] encontraron altos arroyo Tinajas que contaminó el río Bacanuchi, en el 2014) y de las lagunas sobre los terreros de jales que pueden contaminar fácilmente al Río Sonora.

5.El lixiviado de minas de oro abandonadas puede ser fuente de contaminación por arsénico, compuesto altamente tóxico. Aunque el arsénico forma parte de la geología de Sonora, la extracción y fundición de metales y combustión de carbón puede aumentar su grado de contaminación [10].

6. En el río San Miguel, por otra parte, también se ha analizado la presencia de tóxicos, encontrándose valores de arsénico, bario, cadmio, cromo, hierro, plomo, manganeso, molibdeno, níquel, selenio, estroncio, titanio, vanadio y zinc que exceden a su vez los límites máximos permisibles para el consumo humano [4].

7.La CRS posee algunas minas menores inactivas abandonadas por mineros de pequeña escala, 


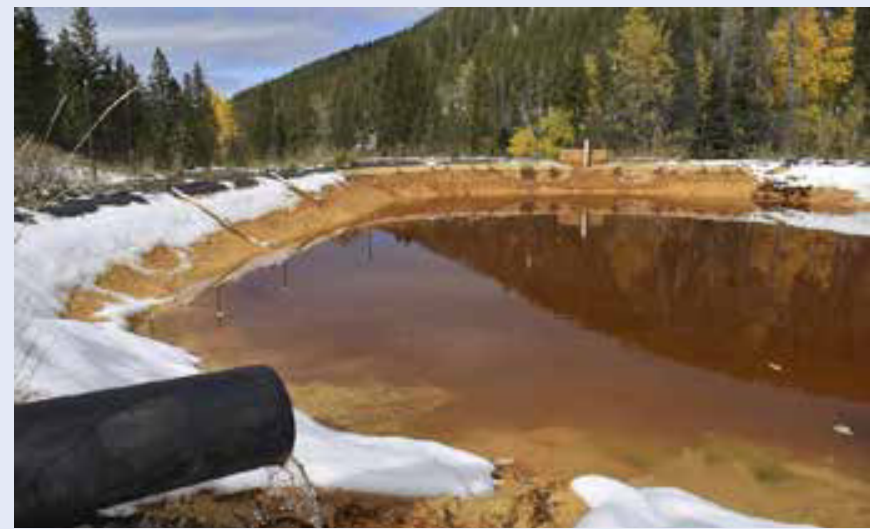

especialmente a los alrededores del río San Miguel y el Río Sonora. Guzmán et al. (2019) [11] sin embargo, analizaron recientemente sedimentos de diferentes sitios de la CRS cercanos a minas menores inactivas y demostraron que no hay un impacto de estas minas en la calidad de agua de las corrientes de la cuenca, esto debido a que las concentraciones de metales liberadas por derrames esporádicos por este tipo de minas son sustancialmente menores a liberaciones de minas en operación en la misma cuenca. A pesar de que minas menores abandonadas no han mostrado generar gran impacto ambiental en las aguas superficiales según dichos autores, la gran cantidad de mineros gambusinos activos sí podrían generar un impacto en la contaminación de las cuencas.

8. En el caso de la minería a pequeña escala, el mercurio y el cianuro son las principales sustancias usadas para extraer oro. El mercurio específicamente se usa para la amalgamación y obtención del oro y suele ser liberado a las corrientes de agua, donde forma metilmercurio, que se acumula fácilmente en seres vivos como los peces y los animales que los consuman, incluyendo al humano, causando severos daños en la salud [12].

9. En la CRY, las principales minas activas con mayor potencial de contaminar las aguas superficiales son la mina de cobre más grande de México, La Caridad, y las minas de oro La India y Mulatos, todas localizadas cerca de afluentes al río Yaqui, siendo su proceso de extracción de cobre y oro también por medio de lixiviación. En Nacozari de García se encuentra además

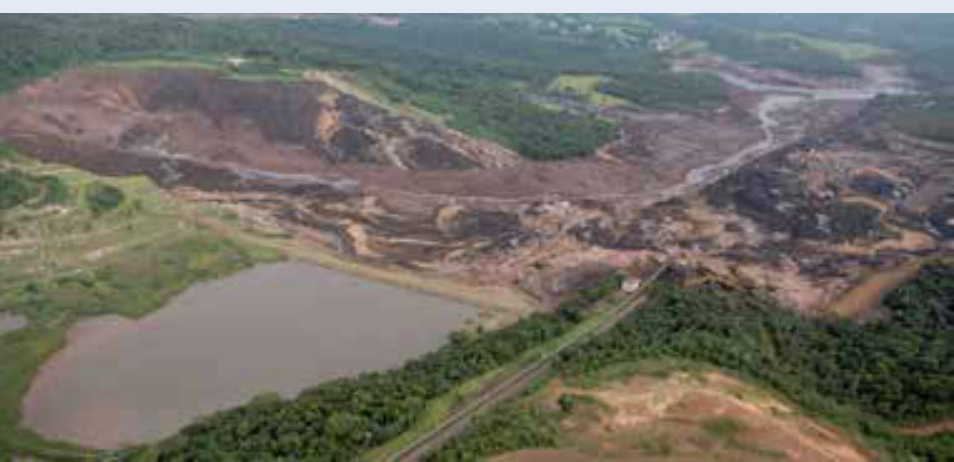

una planta fundidora perteneciente a Grupo México, formada por una fundición que tuesta concentrados de cobre produciendo cobre blíster, una refinadora que produce cobre electrolítico y una fábrica de ácido sulfúrico que también pueden llegar a contaminar las aguas superficiales. Más al sur, en Cumpas se encuentra también la planta metalúrgica Molymex que produce trióxido de Molibdeno [1].

10. El municipio de Nacozari de García contiene terreros de jales dejados desde principios del siglo pasado por la antigua concentradora que procesó materiales provenientes de minas como la de Pilares de Nacozari. Estos jales acumulan más de 20 millones de toneladas de desechos mineros, que, por su inestabilidad, contenido de tóxicos como el arsénico y lo expuestos que están hacia la población pueden causar daños ambientales y daños a la salud si ingresaran por el Río Nacozari, afluente del río Moctezuma, que desemboca en la presa del Novillo. Se ha caracterizado la composición química de los jales de Nacozari, encontrándose la presencia de elevadas concentraciones de hierro y cobre, así como además la baja presencia de otros metales como arsénico, calcio, cadmio, plomo, zinc, antimonio, bismuto, molibdeno y cromo [13]. Varios estudios han evaluado la contaminación de los jales de Nacozari de García, entre ellos Mendoza-Ibarra (2013) [10] que usó la metodología de la EPA para evaluaciones preliminares de riesgos como en el presente estudio, obteniendo como resultado que el sitio es potencialmente peligroso, especialmente para el Río Nacozari.

En la Figura 1 se presenta la ubicación, actividad, y principales minerales extraídos de todas las minas analizadas que se incluyeron en el Archivo de Sitios Identificados generado por la información de la revisión bibliográfica.

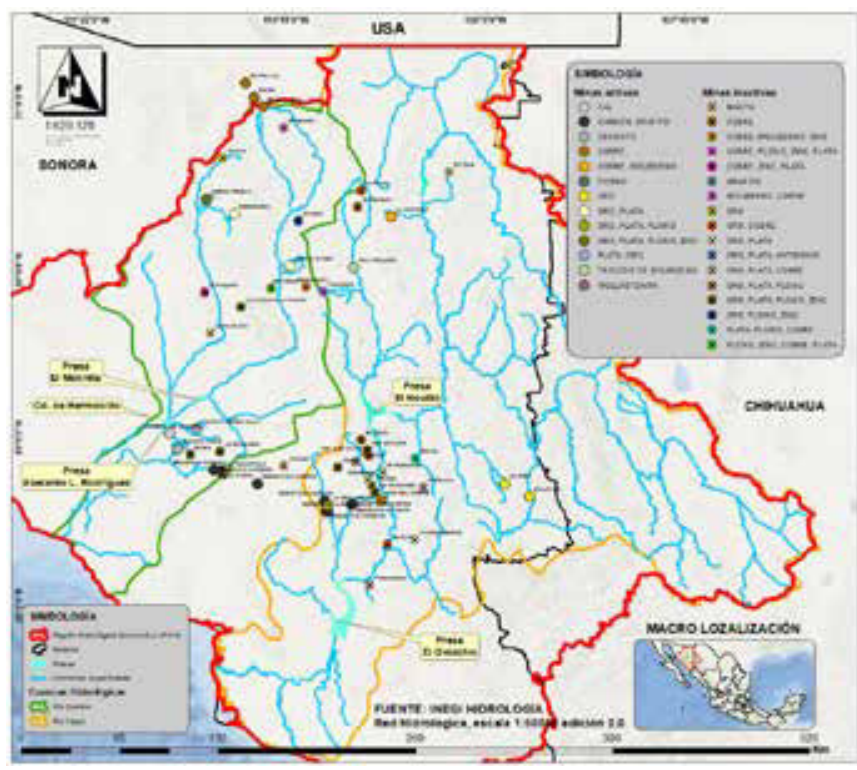

Figura 1. Minas de la Región Hidrológica Sonora-Sur 
Finalmente, la metodología de clasificación de sitios dio los siguientes resultados:

1. Permitió disminuir el número de sitios que se deben estudiar quedando los enlistados en la Tabla 1.

\section{Tabla 1. Ponderación de Peligros (PP) de las minas analizadas}

\begin{tabular}{|l|l|c|c|}
\hline Cuenca & \multicolumn{1}{|c|}{ Mina } & $\begin{array}{c}\text { Activa (A) o } \\
\text { Inactiva (I) }\end{array}$ & PP \\
\hline & Planta Hermosillo & A & 32 \\
\hline Calhidra & A & 32 \\
\hline Planta Yaqui & A & 32 \\
\hline \multirow{2}{*}{ Río Sonora } & Beneficia Carbón & A & 32 \\
\hline & Buenavista & A & 100 \\
\hline Amelia & I & 100 \\
\hline Cerro Prieto & A & 100 \\
\hline Mercedes & A & 100 \\
\hline Santa Elena & A & 100 \\
\hline Moly-Planta & A & 32 \\
\hline México & I & 32 \\
\hline Beneficia Carbón & A & 32 \\
\hline La Caridad & A & 100 \\
\hline Cumobabi & I & 100 \\
\hline Mulatos & A & 100 \\
\hline La India & A & 100 \\
\hline Luz del Cobre & A & 100 \\
\hline La Bonancita & A & 100 \\
\hline Pilares & I & 100 \\
\hline
\end{tabular}

\section{CONCLUSIONES}

" La metodología utilizada en este estudio para ponderar peligros de contaminación de ríos por derrames minero-metalúrgicos fue útil para seleccionar las minas que presentan mayor riesgo de contaminación a las corrientes superficiales de la Región Hidrológica Sonora Sur. Este método permite disminuir el número de sitios analizados hasta obtener los que presentan un más probable riesgo de contaminar las aguas superficiales. Por lo tanto, el uso de esta metodología también puede servir como screening previo a la inspección de sitio.

Las minas activas que presentan una mayor posibilidad de producir contaminaciones del agua superficial, con una PP de 100, son Buenavista del Cobre, La Caridad,
Cerro Prieto, Mercedes, Santa Elena, La India, Mulatos, la Bonancita, Luz del Cobre

Las minas inactivas con probabilidad de contaminación de aguas superficiales son: Pilares, Cumobabi y Amelia.

»Instalacionesminero-metalúrgicosqueseconsideraron como fuentes potenciales de contaminación de agua, con una PP de 32, fueron minas de carbón y plantas productoras de cemento y cal.

\section{BIBLIOGRAFIA}

[1] Secretaría de Economía del Estado de Sonora (2017). "Panorama Minero del Estado de Sonora". Gobierno del Estado de Sonora.

[2] Vega-Granillo, E. L., Cirett-Galán, S., De la Parra-Velasco, M. L. y Zavala-Juárez, R. (2011). "Hidrogeología de Sonora, México" en Panorama de la geología de Sonora, México: Universidad Nacional Autónoma de México, Instituto de Geología, Boletín 118, Calmus, Thierry, ed., p. 267-298.

[3] Ley de Aguas Nacionales. 2016. Diario Oficial de la Federación, 1 de diciembre de 1992. Última reforma publicada el 24 de marzo de 2016. Ciudad de México, México.

[4] Moreno, S. A. y Rodrígez J.M. (2017). "Metodologías de análisis ambiental y valoración de los efectos de la minería en el entorno global". Epistemus: 26-33.

[5] León-García, G. L., Meza-Figueroa, D. M., Valenzuela-García, et al. (2018). "Study of heavy metal pollution in arid and semiarid regions due to mining activity: Sonora and Bacanuchi rivers". Int. J. Environ. Sci. Nat. Res. 11(1):555804.

[6] Jaishankar, M., Tseten, T., Anbalagan, N., Mathew, B. B. y Beeregowda, K. N. (2014). "Toxicity, mechanism and health effects of some heavy metals". Interdiscip. Toxicol. 7(2), 6072. https://doi.org/10.2478/intox2014-0009.

[7] U.S. Environmental Protection Agency (EPA) (1991). "Guidance for Performing Preliminary Assessments Under CERCLA". Office of Emergency and Remedial Response.

[8] Gómez-Álvarez, A., Villalba-Atondo, A., Acosta-Ruiz, G., Castañeda-Olivares, M. y Kamp, D. (2004). "Metales pesados en el agua superficial del Río San Pedro durante 1997 y 1999. Rev. Int. Contam. Ambient 20(1): 5-12.

[9] Villalba-Atondo A. y Gómez-Álvarez A. (2011). Calidad del agua en la región noroeste del estado de Sonora, México. Ed. Académica Española.

[10] Mendoza-Ibarra E. (2013) “Evaluación preliminar de riesgos de la salud humana por contaminación por desechos mineros del agua superficial en la subcuenca Río Moctezuma en Nacozari de García, Sonora". Tesis de licenciatura. Universidad de Sonora.

[11] Guzmán, H. M., Gómez-Álvarez, A., Valenzuela-García, J. L., Encinas-Romero, M. A., Villalba-Atondo A. I., Encinas-Soto K. K. (2019). "Assesment of the impacto on sediment quality from abandoned artisanal mine runoffs in a semi-arid environment (the Sonora River basin- Nortwestern Mexico. Environ. Earth Sci. 78:145.

[12] Spiegel, S. (2009). "Socioeconomic dimensions of mercury pollution abatement: Engaging artisanal mining communities in Sub-Saharan Africa." Ecol Econ. 68(12): 30723083.

[13] Alvarado-Martínez, V. J., Volke-Sepúlveda, T. L., SalgadoFigueroa. P., De la Rosa-Pérez, A. (2004) Informe de resultados del proyecto: Método de análisis y propuesta para el manejo de los residuos mineros del sitio de Nacozari, Sonora. Dirección General del Centro Nacional de Investigación y Capacitación Ambiental. Secretaría de Medio Ambiente y Recursos Naturales - Instituto Nacional de Ecología. 